\title{
Finite Element Analysis of Proximal Cement Fixation in Total Hip Arthroplasty
}

\author{
Maizatul Afirah Ahmad ${ }^{1,3}$, Nurul Nadhirah Mohamed Elias Zulkifli' ${ }^{1}$, Solehuddin Shuib ${ }^{1}$, \\ Shahrul Hisham Sulaiman ${ }^{2}$, Abdul Halim Abdullah ${ }^{1^{*}}$ \\ ${ }^{1}$ Faculty of Mechanical Engineering, Universiti Teknologi MARA, 40450 Shah Alam, Selangor, Malaysia \\ ${ }^{2}$ Faculty of Medicine, Universiti Teknologi MARA, Sungai Buloh Campus, 47000 Sungai Buloh, Selangor, \\ Malaysia \\ 33D Gens Sdn. Bhd., 18 Jalan Kerawang U8/108, Perindustrian Tekno Jelutong, Seksyen U8, 40150 Shah \\ Alam, Selangor, Malaysia
}

\begin{abstract}
Total hip arthroplasty (THA), or surgical replacement of the hip joint with a prosthesis, is a reconstructive procedure that has improved the management of hip joint diseases that have responded poorly to conventional medical therapy. There are reasons to believe that the proximal part of the cement withstands more stress than the distal part in THA. Therefore, this study aims to determine whether it is possible to perform THA by cementing only the proximal part of the hip prosthesis. The polymethylmethacrylate cement has a Young's modulus of $2 \mathrm{GPa}$, a tensile strength of $29 \mathrm{MPa}$, and a Poisson's ratio of 0.3. This analysis was done using a stainless steel stem model provided by the Department of Orthopaedic Surgery, University of Malaya Medical Centre, using a Young's modulus of $200 \mathrm{GPa}$ and a Poisson's ratio of 0.28 . The bone cement was modelled while the THA femur was reconstructed by inserting stem prosthesis into the femoral canal. The effects of different proximal cement lengths in THA were investigated by analyzing the stress distribution and displacement of the THA model during walking and stair climbing.
\end{abstract}

Keywords: Cemented THA; Finite element analysis; Proximal cementation; Total hip arthroplasty

\section{Introduction}

The number of patients who have undergone total hip arthroplasty (THA) is continually increasing. The most common indicators for primary THA are osteoarthritis, particularly for older patients (aged 75 years and over), avascular necrosis, rheumatoid arthritis, developmental dysplasia of the hip, and osteoporosis (Amanatullah et al., 2010). Normally, these patients can barely walk and experience continuous pain in their daily lives. THA is believed to help increase quality of life and improve joint function, allowing older patients to function normally and younger patients to resume sports activities. During THA, a surgeon makes an incision over the head and proximal neck of the femur and removes layers of the hip socket. Then, a metal ball and stem are inserted in the femur and a plastic socket is placed in the enlarged pelvis cup. To obtain successful results, these components must be fixed firmly to the bone, either with polymethylmethacrylate cement or a cementless fixation, via bone ingrowth into a porous surface, resulting in biologic fixation (Choi, 2015). 
THA is believed to be the greatest advancement in surgery in the second half of the $20^{\text {th }}$ century. In the late 1940s, researchers experimented with and developed many different materials, surgical techniques, fixation methods, and implant design. Sir John Charnley attempted to design an artificial hip joint using biomechanical principles of human hip joint function (Abdullah et al., 2010). The outcome yielded very low friction bearing surfaces that helped reduce friction and wear rates, resulting delayed aseptic loosening. Several aspects marked the prosthesis as successful, namely longevity, ease of implantation, and revisability.

Many factors need to be focused on, such as implant design, materials, and fixation. The fixation method can be cemented or uncemented. Cemented methods were widely used in early procedures and have gained popularity since being introduced by John Charnley in 1972; they have been continuously improved throughout the decades (Abdullah et al., 2010). They use polymethylmethacrylate (PMMA), or bone cement, to affix the prosthesis to the bone. Prior to surgery, the femoral canal is injected with bone cement to secure the prosthesis in its ideal position.

The type of fixation method used depends on the patient's age. For example, cementless fixation is favored for young and active patients. Though many studies have been conducted on cemented and cementless hip arthroplasty (Abdullah et al., 2017; Zhang et al., 2017; Todo, 2018), no studies have specifically focused on proximal cementation fixation in fully cemented implants and the effects of bone cement length. Einsiedel et al. (2008) reported the advantages of proximal hip stem fixation based on their findings using the new Z-shaft implant. It was a partially cemented stem, known as a hybrid model, with proximal cementation and cranial press fit. Similar studies describing the performance of proximal fixation referred to the hybrid stem model (Pennington et al., 2013; Samra and Paliobeis, 2015; Valle et al., 2016; Wangen et al., 2017; Zhang et al., 2017; Jonas et al., 2019; Nawfal et al., 2020). In this study, the proposed proximal fixation was applied to the fully cemented implant model. We expect to improve existing cemented stem fixation by minimizing the usage of bone cement. Hence, this study will predict the optimum cement length and the effects of different cement lengths in cemented hip arthroplasty by analyzing stress distribution and displacement.

\section{Methods}

Femoral bone geometry will be developed using computed tomography (CT) images of cadavers provided by the University of Malaya Medical Centre (UMMC). CT scan data are very helpful in modern medicine and are also essential in solving biomedical-related complications (Kim et al., 2013). To render an accurate 3D model, high-resolution CT scan data with a slice thickness less than $2 \mathrm{~mm}$ were required. The CT images were compiled in a DICOM application and stacked in commercial biomedical software (Mimics) to develop inhomogeneous 3D finite element models. Each element of the bone model was generated based on the linear relationship between apparent density and gray density values using the Hounsfield scale (Bessho et al., 2009; Abdullah et al., 2017). The data appeared in slices with three different plane views: axial, coronal, and sagittal (Amir Shahlan et al., 2017). This helps obtain more accurate information from the CT data. Several parameters in biomedical software were used to ensure that the 3D femur model was correct.

The analysis focused on the upper half of the femoral bone instead of the full femur to reduce the analysis time. The Exeter Stem (provided by the Department of Orthopaedic Surgery, University of Malaya Medical Centre) was used in this analysis because it is known for its flexibility and ability to reduce cement damage accumulation. A cement mantle with a thickness of $3 \mathrm{~mm}$ was used to complete the THA model, which was then analyzed using 
Abaqus ver. 2018, a commercial finite element software. Finite element analysis (FEA) is recognized as one of the most cost-effective methods for predicting optimal design and product performance (Kurdi et al., 2010).

Cemented hip arthroplasty models are constructed by replacing the hip joint with femoral component models, which consist of a stem prosthesis, a femoral ball, liner, and an acetabular cup. The femoral head was cut off and the stem prosthesis was aligned appropriately in the femoral canal. Several bone cement models were used to represent different types of proximal bone cement fixation. In addition to full proximal cementation, in this study, we proposed the use of proximal cementation with reductions of $40 \mathrm{~mm}, 80$ $\mathrm{mm}$, and $120 \mathrm{~mm}$, as illustrated in Figure 1. To the authors' knowledge, to date, no study on proximal cemented techniques has been conducted. The three levels of proximal cement proposed in this study will serve as pioneer findings in this field.

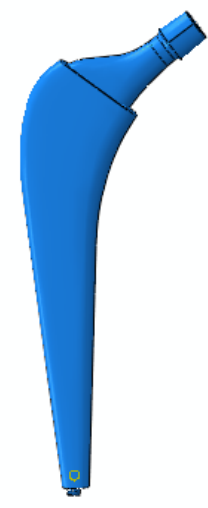

(a)

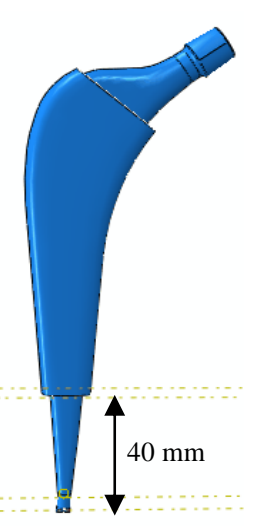

(b)

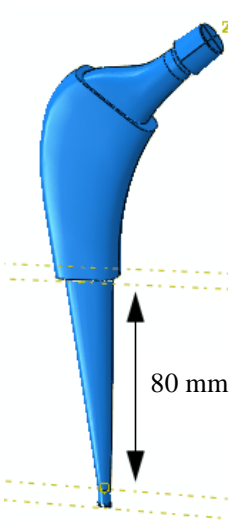

(c)

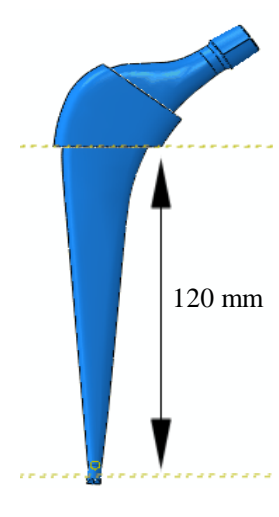

(d)

Figure 1 The types of proximal cementation used in this study: (a) fully cemented; (b) $40 \mathrm{~mm}$ cut off; (c) $80 \mathrm{~mm}$ cut off; and (d) $120 \mathrm{~mm}$ cut off

\subsection{Material Properties}

The stem prosthesis was built from a stainless steel material widely used in bone implants; it has excellent corrosion resistance and high strength (Jujur et al., 2015). In this study, a reverse engineering process was applied to capture the stem component. The femoral bone was consisted of cancellous bone and a cortical shell. In this case, polymethyl methacrylate (PMMA) was assigned as the bone cement. The material properties for all models were simulated to be isotropic and homogenous. All assigned material properties were computed using the survey done by Lamvohee et al. (2014). Table 1 shows the material properties for each element.

Table 1 Material properties used in the FEA model

\begin{tabular}{clccc}
\hline No. & Material & $\begin{array}{c}\text { Young } \\
\text { Modulus, } \\
\text { E (GPa) }\end{array}$ & $\begin{array}{c}\text { Poisson's } \\
\text { Ratio }\end{array}$ & $\begin{array}{c}\text { Yield Strength } \\
\text { (MPa) }\end{array}$ \\
\hline 1 & Femoral Bone & 17 & 0.3 & 115 \\
2 & PMMA & 2 & 0.3 & 29 \\
3 & Stem Prosthesis & 200 & 0.28 & 205 \\
4 & Distal Centralizer & 2 & 0.3 & 29 \\
\hline
\end{tabular}




\subsection{Meshing}

The reconstructed model consisted of hollow femoral bone, a stem prosthesis, a distal centralizer (stopper), and a cement mantle meshed using commercial finite element software. A four-node tetrahedral element with a mesh size of $2 \mathrm{~mm}$ was used to discretize the model, as shown in Figure 2. This element is commonly used as it can be simulated and meshed with almost any volume despite its size and complexity; it is also versatile in contact analysis (Wittek and Miller, 2020). The number of elements for all models were summarized in Table 2 .

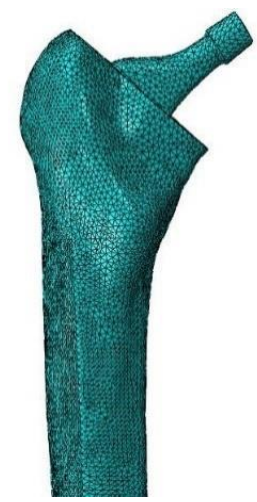

(a)

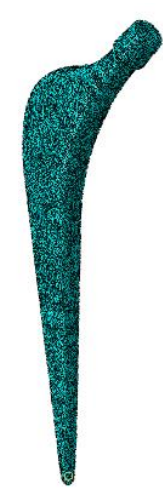

(b)

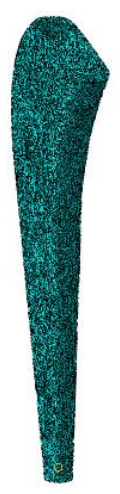

(c)

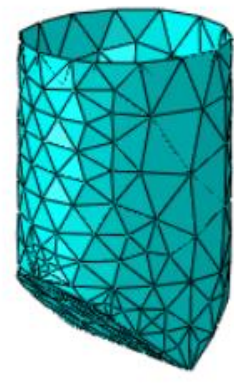

(d)

Figure 2 FEA mesh models for: (a) the THA femur; (b) the stem prosthesis; (c) the bone cement mantle; and (d) the distal centralizer (stopper)

Table 2 Number of elements in each cement model.

\begin{tabular}{cccc}
\hline \multirow{2}{*}{ THA Model } & \multicolumn{3}{c}{ Number of Elements } \\
\cline { 2 - 4 } & Femur & Stem Prosthesis & Cement Mantle \\
\hline Fully Cemented & 279,923 & 30,256 & 18,764 \\
40 mm cut off & 92,360 & 11,337 & 6,277 \\
80 mm cut off & 92,360 & 11,337 & 4,650 \\
120 mm cut off & 92,360 & 11,337 & 1,950 \\
\hline
\end{tabular}

Table 3 Magnitude and direction of hip joint contact and abductor force in walking and stair climbing

\begin{tabular}{ccccc}
\hline Point & Forces & $\mathrm{x}$ & $\mathrm{y}$ & $\mathrm{z}$ \\
\hline \multicolumn{5}{c}{ Walking } \\
\hline P1 & Hip Joint Contact & -263.8 & -1841.3 & -433.8 \\
P2 & Abductor & 34.5 & 695 & 465.9 \\
\hline \multicolumn{5}{c}{ Stair Climbing } \\
P1 & Hip Joint Contact & -486.8 & -1898.3 & -476.4 \\
P2 & Abductor & 231.4 & 682.1 & 563.1 \\
\hline
\end{tabular}




\subsection{Load and Boundary Conditions}

Physiological loads, namely stair climbing and walking, were considered in the analysis to predict the effects of proximal cementation fixation (Simões et al., 2000). The results of stress-strain distribution and displacement were analyzed to predict bone adaptation and implant stability in proximal cementation. The reconstruction model used a femoral bone, a stem prosthesis, and a cement mantle. The load magnitude of the model is shown in Table 3 (Abdullah et al., 2010).

\section{Results and Discussion}

\subsection{Stress Distribution along the Cemented Hip Arthroplasty Model}

The effects of proximal cement fixation were observed in the resulting von Mises stress and total displacement values. Maximum magnitudes were measured and compared for all models to determine potential effects.

Figure 3 shows the stress distribution along the intact femoral bone and the cross section of various THA models during stair climbing. Results for walking activities indicated similar pattern of stress distribution but in lower magnitude. Yet, the stress distribution of the stair climbing activity was presented in this paper. However, there is a significant difference in the distribution of stress in the femoral bone and THA models (Goshulak et al., 2016). Stress is more dominant at the prosthesis stem in the THA model than in the intact femur. Less stress in the THA femur in the implant's surrounding areas may lead to the stress shielding phenomenon. The highest von Mises stress value during walking is 115 $\mathrm{MPa}$; during stair climbing, it is $205 \mathrm{MPa}$. In conjunction with the bending action of the abductors, the moment of the proximodistal element causes medial bending with lateral tensile stress and compressive stress on the medial area (Jonkers et al., 2008; Todo, 2018). High stress is often correlated with the boundary condition that has been set up and calculated at the end of the model.

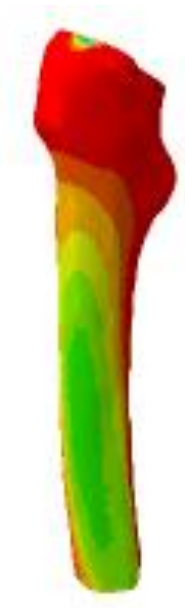

(a)

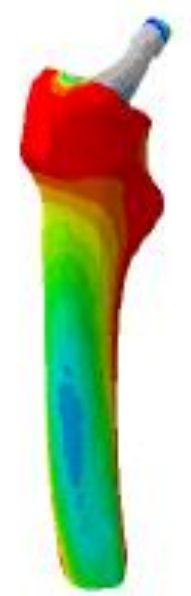

(b)

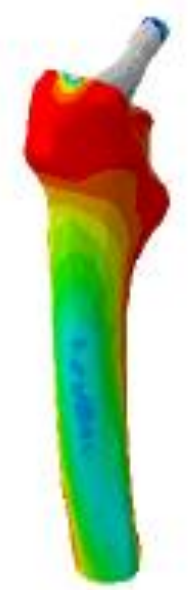

(c)

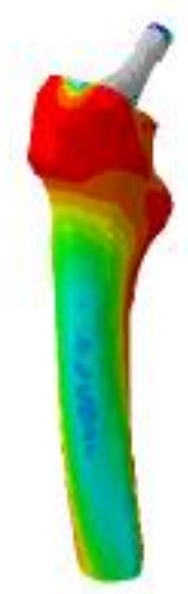

(d)

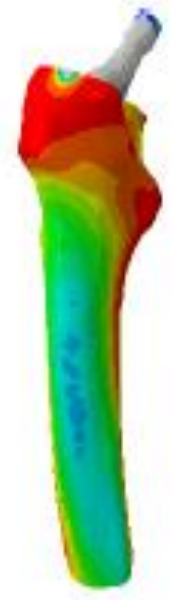

(e)

Figure 3 Stress distribution for cemented THA at: (a) half femur; (b) fully cemented; (c) $40 \mathrm{~mm}$ cut off; (d) $80 \mathrm{~mm}$ cut off; and (e) $120 \mathrm{~mm}$ cut off

Stress distribution within bone cement is presented in Figure 4. The maximum stress exhibited during walking and stair climbing was $14.3 \mathrm{MPa}$ and $14.82 \mathrm{MPa}$, respectively. This also demonstrates that force applied to the cement prevents stem prosthesis displacement. These findings are consistent with Aznan et al. (2020). The difference in maximum stress magnitude between the two studies was expected due to different bone models, loading 
magnitudes, and positions. The maximum stress is predicted at the upper part of the bone cement suggested for the importance of proximal fixation to strengthen the stem prosthesis stability. Proximal cemented techniques help the load transmission to the proximal femur region and manage to protect the distal bone in the case of revision (Einsiedel et al., 2008). Different lengths can be considered for patients whose bone quality is not sufficient to obtain primary stability through the press fit technique.

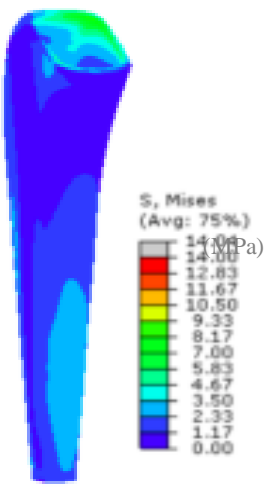

(a)

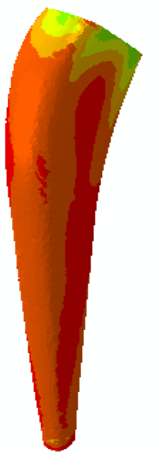

(b)

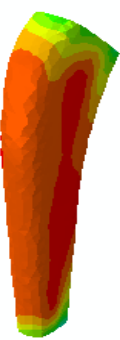

(c)

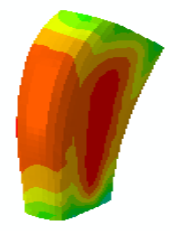

(d)

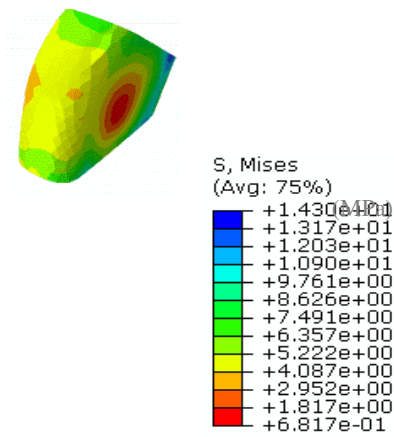

(e)

Figure 4 Stress variation results for bone cement: (a) full cement (Aznan et al., 2020); (b) full cement; (c) $40 \mathrm{~mm}$ cut off; (d) $80 \mathrm{~mm}$ cut off; and (e) $120 \mathrm{~mm}$ cut off

\subsection{Effects of Total Displacement in Stem Prosthesis and Cement Mantle}

Figures 5 and 6 show the total displacement distribution of the stem prosthesis and cement mantle, respectively, for stair climbing. Maximum magnitudes of $3.28 \mu \mathrm{m}$ and 3.38 $\mu \mathrm{m}$ can be seen at the top of the cement for walking and stair climbing, respectively. This could be due to the fact that low amounts of cement yield a high concentrated force on the stem prosthesis. The high magnitude of stress micromotion at the tip of the cement mantle caused by bending will cause cracking to occur. PMMA serves as the interphase between the spongy bone and the rigid prosthesis. The results showed that the maximum stress was located at the neck section and at the distal end of the prosthesis.

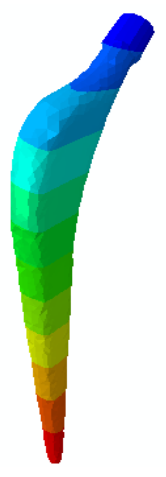

(a)

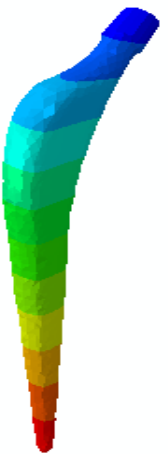

(b)

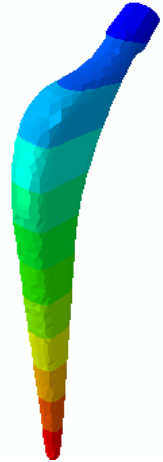

(c)

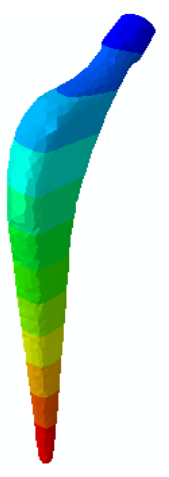

(d)

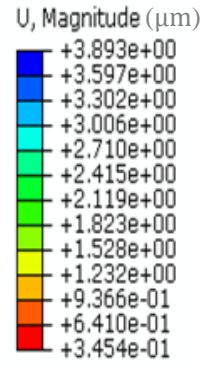

Figure 5 Displacement of stem prosthesis during stair climbing for: (a) fully cemented; (b) $40 \mathrm{~mm}$ cut off; (c) $80 \mathrm{~mm}$ cut off; and (d) $120 \mathrm{~mm}$ cut off models 


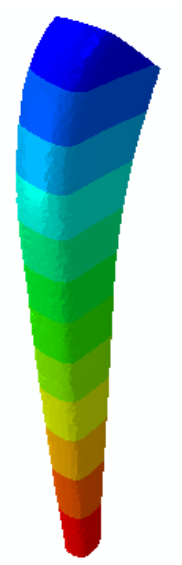

(a)

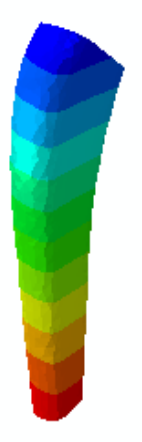

(b)

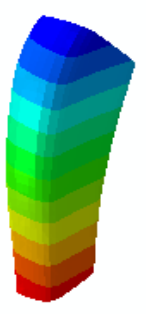

(c)
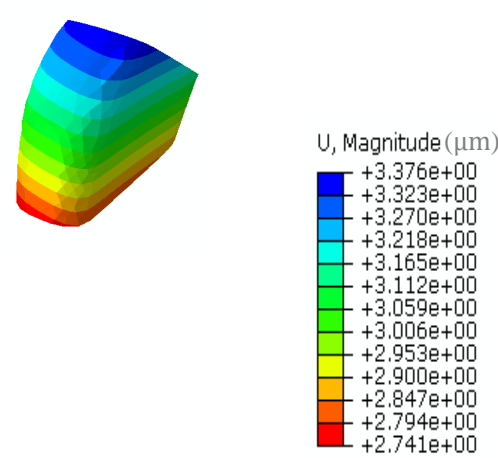

(d)

Figure 6 Displacement of cement mantle during stair climbing for: (a) fully cemented; (b) $40 \mathrm{~mm}$ cut off; (c) $80 \mathrm{~mm}$ cut off; and (d) $120 \mathrm{~mm}$ cut off models

Figure 7 compares the highest total displacement of the cement mantle during walking and stair climbing. The maximum displacement recorded was $3.88 \mu \mathrm{m}$ for walking and 3.89 $\mu \mathrm{m}$ for stair climbing. Both maximum displacements are located in the $120 \mathrm{~mm}$ cut off model because it has the least contact with the cement. This model has a higher deform rate than the other three models. The maximum von Mises stress values for walking and stair climbing were $140 \mathrm{MPa}$ and $157.50 \mathrm{MPa}$, respectively. Based on previous studies, debonding usually occurs in high stress areas (Abdullah et al., 2010; Aznan et al., 2020). A higher magnitude was expected for stair climbing than walking.

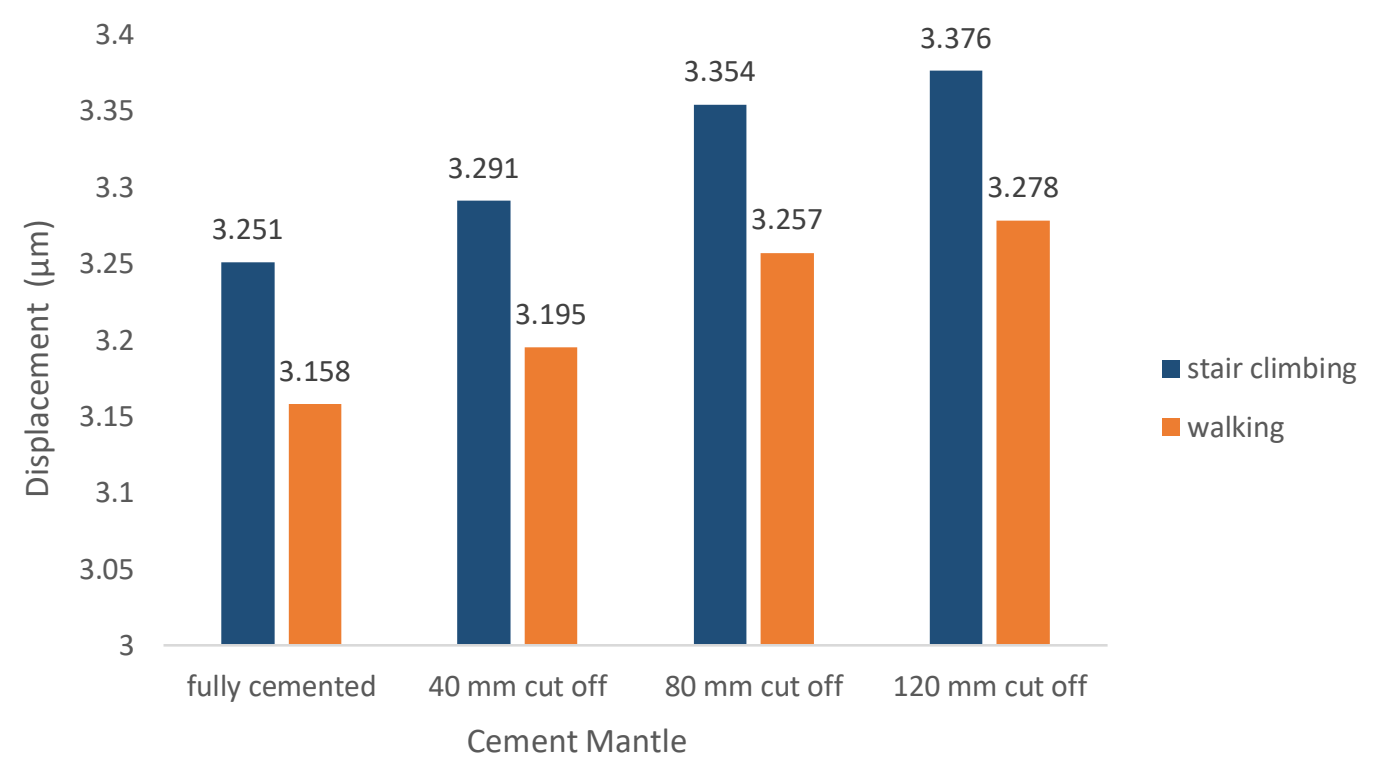

Figure 7 Comparing maximum displacement values for different cement mantle models

A small increase in total displacement for the stem and cement mantle paired with an increase in the proximal cement cut off suggests a risk of implant instability. The excessive micromotion will drive fibrous encapsulation and implant loosening, which could affect the tissue healing response (Wazen et al., 2013). The maximum displacement is predicted at 
the proximal region of the prosthesis and the cement mantle. The hip loading applied to the prosthesis stem will lead to bending effects and further contribute to higher movement at the proximal region of the prosthesis. The existing abductor muscle will minimize the effects. The maximum load transfers occurred at the distal end of the prosthesis, minimizing the micromotion at the respective region (Amirouche et al., 2016). Our findings suggest that the fully cemented technique will provide minimum displacement and further minimize the risk of implant loosening. The small differences in maximum displacement for all models suggested minimal effects.

\section{Conclusions}

The stress-strain distribution and displacement of different cemented THAs during stair climbing and walking were successfully analyzed using FEA. The results showed that the von Mises stress value did not exceed the yield strength, which were $115 \mathrm{MPa}$, $205 \mathrm{MPa}$, and $29 \mathrm{MPa}$, for femoral bone, stem prosthesis and bone cement, respectively. Yield strength is the stress at which a material begins to deform plastically, while yield point is when non-linear deformation begins. Hence, no models were permanently deformed. The highest displacement values for the cement mantle were $3.376 \mu \mathrm{m}$ and $3.278 \mu \mathrm{m}$ for stair climbing and walking activities, respectively. The total displacement increment of the stem and cement mantle with increasing proximal cement cut off suggests the risk of implant loosening at higher cement cut offs.

\section{Acknowledgements}

This research was supported by Universiti Teknologi MARA, UiTM under Grant No. 600-IRMI/PERDANA 5/3 BESTARI (103/2018). We thank and acknowledge the Ministry of Higher Education in Malaysia, our colleagues from the Department of Orthopaedic Surgery, University of Malaya Medical Centre, and the UiTM Penang Branch for their computational software facilities and for providing insight and expertise in the research work.

\section{References}

Abdullah, A.H., Mohd Nor, M.A., Saman, A.M., Tamin, M.N., Abdul Kadir, M.R., 2010. Effects of Prosthesis Stem Tapers on Stress Distribution of Cemented Hip Arthroplasty. In: AIP Conference Proceedings, Volume 1285, pp. 561-575

Abdullah, A.H., Todo, M., Nakashima, Y., 2017. Prediction of Damage Formation in Hip Arthroplasties by Finite Element Analysis using Computed Tomography Images. Medical Engineering and Physics, Volume 44, pp. 8-15

Amanatullah, D.F., Cheung, Y., Di Cesare, P.E., 2010. Hip Resurfacing Arthroplasty: A Review of the Evidence for Surgical Technique, Outcome, and Complications. Orthopedic Clinics of North America, Volume 41(2), pp. 263-272

Amirouche, F., Solitro, G., Walia, A., 2016. No Effect of Femoral Offset on Bone Implant Micromotion in an Experimental Model. Orthopaedics \& Traumatology: Surgery \& Research, Volume 102(3), pp. 379-385

Amir Shahlan, M.A.M., Nasrul Anuar, A.R., Abdul Kadir, M.R., Hadi, M.Y., 2017. Development of 3-Dimensional Model of Femur Bone Considering Cortical and Cancellous Structures. International Journal of Engineering Technology and Sciences, Volume 7(1), pp. 1-8

Aznan, N., Yusof, M.S., Sulaiman, S.H., Todo, M., Abdullah, A.H., 2020. Effects of Retroversion and Anteversion Alignment in Cemented Hip Arthroplasty. Journal of Mechanical Engineering, Volume 9(1), pp. 25-41 
Bessho, M., Ohnishi, I., Matsumoto, T., Ohashi, S., Matsuyama, J., Tobita, K., Kaneko, M., Nakamura, K., 2009. Prediction of Proximal Femur Strength using a CT-based Nonlinear Finite Element Method: Differences in Predicted Fracture Load and Site with Changing Load and Boundary Conditions. Bone, Volume 45(2), pp. 226-231

Choi, S., 2015. Total Hip Arthroplasty. In: Decision-Making in Orthopedic and Regional Anesthesiology: A Case-based Approach, Cambridge University Press, Cambridge, UK, pp. 95-100

Einsiedel, T., Gebhard, F., Bregolato, I., Hiemeier, A., Kinzi, L., Schultheiss, M., 2008. Proximal Cement Fixation in Total Hip Arthroplasty - First Results with a New Stem Design. International Orthopaedics, Volume 32(3), pp. 295-306

Goshulak, P., Samiezadeh, S., Aziz, M.S.R., Bougherara, H., Zdero, R., Schemitsch, E.H., 2016. The Biomechanical Effect of Anteversion and Modular Neck Offset on Stress Shielding for Short-Stem Versus Conventional Long-Stem Hip Implants. Medical Engineering and Physics, Volume 38(3), pp. 232-240

Jonas, S.C., Whitehouse, M.R., Bick, S., Bannister, G.C., Baker, R.P., 2019. An 18-Year Comparison of Hybrid Total Hip Replacement and Birmingham Hip Resurfacing in Active Young Patients. HIP International, Volume 29(6), pp. 630-637

Jonkers, I., Sauwen, N., Lenaerts, G., Mulier, M., Perre, G. Van Der, Jaecques, S., 2008. Relation between Subject-Specific Hip Joint Loading, Stress Distribution in the Proximal Femur and Bone Mineral Density Changes after Total Hip Replacement. Journal of Biomechanics, Volume 41(16), pp. 3405-3413

Jujur, I.N., Sah, J., Bakri, A., Wargadipura, A.H.S., 2015. Analysis of Oxide Inclusions on Medical Grade 316L Stainless Steel using Local Raw. International Journal of Technology, Volume 6(7), pp. 1184-1190

Kim, S.C., Jung, H.M., 2013. A Study on Performance of Low-Dose Medical Radiation Shielding Fiber (RSF) in CT Scans. International Journal of Technology, Volume 4(2), pp. 178-187

Kurdi, O., Rahman, R.A., 2010. Finite Element Analysis of Road Roughness Effect on Stress Distribution of Heavy-Duty Truck Chassis. International Journal of Technology, Volume 1(1), pp. 57-64

Lamvohee, J.M.S., Ingle, P., Cheah, K., Dowell, J., Mootanah, R., 2014. Total Hip Replacement: Tensile Stress in Bone Cement is Influenced by Cement Mantle Thickness, Acetabular Size, Bone Quality, and Body Mass Index. Journal of Computer Science \& Systems Biology, Volume 7(3), pp. 72-78

Nawfal, N., Karthik, M.N., Satish K.C., 2020. Reverse Hybrid Total Hip Arthroplasty: A Good Alternative to Uncemented Total Hip Arthroplasty. International Journal of Orthopaedics Sciences, Volume 6(1), pp. 455-459

Pennington, M., Grieve, R., Sekhon, J.S., Gregg, P., Black, N., van der Meulen, J.H., 2013. Cemented, Cementless, and Hybrid Prostheses for Total Hip Replacement: Cost Effectiveness Analysis. British Medical Journal, Volume 346(f1026), pp. 1-14

Samra, I., Paliobeis, C., 2015. A Dual Biomechanical Failure: Exeter Stem and Pubic Rami Insufficiency Fracture, Following Hybrid Total Hip Arthroplasty. Case Reports in Orthopedics, Volume 2015(2), pp. 1-5

Simões, J.A., Vaz, M.A., Blatcher, S., Taylor, M., 2000. Influence of Head Constraint and Muscle Forces on the Strain Distribution Within the Intact Femur. Medical Engineering and Physics, Volume 22(7), pp. 453-459

Todo, M., 2018. Biomechanical Analysis of Hip Joint Arthroplasties using CT-Image Based Finite Element Method. Journal of Surgery and Research, Volume 1(2), pp. 34-41

Valle, A.G.D., Sharrock, N., Barlow, M., Caceres, L., Go, G., Salvati, E.A., 2016. The Modern, 
Hybrid Total Hip Arthroplasty for Primary Osteoarthritis at the Hospital for Special Surgery. The Bone \& Joint Journal, Volume 98B(1), Suppl A, pp. 54-59

Wangen, H., Havelin, L.I., Fenstad, A.M., Hallan, G., Furnes, O., Pedersen, A.B., Overgaard, S., Karrholm, J., Garellick, G., Makela, K., Eskelinen, A., Nordsletten, L., 2017. Reverse Hybrid Total Hip Arthroplasty: Results from the Nordic Arthroplasty Register Association (NARA). Acta Orthopaedica, Volume 88(3), pp. 248-254

Wazen, R.M., Currey, J.A., Guo, H., Brunski, J.B., Helms, J.A., Nanci, A., 2013. MicromotionInduced Strain Fields Influence Early Stages of Repair at Bone-Implant Interfaces. Acta Biomaterialia, Volume 9(5), pp. 6663-6674

Wittek, A., Miller, K., 2019. Computational Biomechanics for Medical Image Analysis. In S. K. Zhou, D. Rueckert, \& G. Fichtinger (Eds.) In: Handbook of Medical Image Computing and Computer Assisted Intervention, Elsevier, pp. 953-977

Zhang, C.F., Yan, C.H., Zhang, W.M., 2017. Cemented or Cementless Fixation for Primary Hip Arthroplasty-Evidence from the International Joint Replacement Registries. Annals of Joint, Volume 2(57), pp. 1-10 